\title{
Identifying the Pathogen by Multiplex Polymerase Chain Reaction in Bone and Joint Infections: Challenges and Future
}

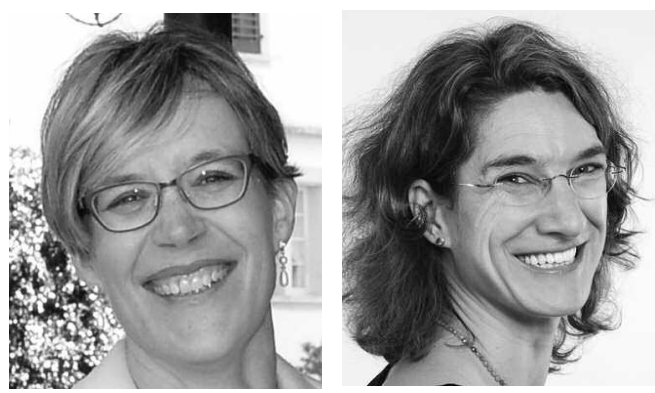

Diagnosis of bone and joint infections remains a challenge. In addition to the difficulty of obtaining sufficient and good clinical samples for microbiological assessment, the bacteria are often few and do not always grow. In these typical biofilm-associated infections, bacteria are embedded in a biofilm matrix, protecting them from the host's immune system as well as from antibiotics used to treat the infection. In addition, biofilm-associated bacteria are difficult to cultivate because they are not easily recovered from the biofilm, they may be few in number, and they are often in a dormant or slow-growing state. To circumvent these difficulties encountered in recovering the bacteria in classical bacterial cultures, some possibilities that have been discussed are PCR, microcalorimetry, and concentrating the recovered fluids. Aiming to broaden the spectrum and efficiency, multiplex PCR assays have been introduced to identify the causative pathogen in bone and joint infections ${ }^{1,2,3,4}$. Because bacterial growth is not required, multiplex PCR have been thought to be the solution for diagnosis of culture-negative orthopedic infections in patients who already took antibiotics prior to the diagnostic investigation. All assays promised to have advantages of a rapid test time and to detect a large number of microorganisms, with specific primers also allowing diagnosis of polymicrobial infections. However, to date no commercial assay has found its way into routine practice, mainly because of low sensitivity or the lack of primers for pathogens not included in the multiplex primers kits.

In this issue of The Journal, Morgenstern, et $a l^{5}$ showed results of a prospective study investigating the role of the multiplex PCR Unyvero implant and tissue infection (ITI) assay. This is a fully automated multiplex PCR aiming to cover over 100 targets, including both pathogens and antibiotic resistance genes.

In 5 out of 14 cases $(35.7 \%)$ with a confirmed culture- positive septic arthritis, the multiplex PCR of the synovial fluid did not identify the pathogen. Among the 10 patients with a positive synovial fluid culture previous to intraoperative diagnostics, 5 (50\%) were PCR-negative. Of these, only 1 patient with a Streptococcus pneumoniae bacteremia had taken antibiotics at the time of diagnosis. In 2 (1 with $S$. pneumoniae, 1 with Clostridium clostridioforme), the pathogen was not detected owing to lack of primers. In the remaining 3 patients (2 Staphylococcus aureus, 1 Streptococcus dysgalactiae), there are no obvious reasons why the PCR failed. Although this study included only a few patients, it clearly showed that the multiplex PCR Unyvero ITI i60 does not improve sensitivity compared to conventional cultures.

\section{Does the new cartridge (ITI G2) overcome the problem of low sensitivity?}

In the new version of the multiplex PCR Unyvero ITI G2, there is an additional primer included for $16 \mathrm{~s}$ rDNA, aiming to circumvent the problem of primers lacking for distinct pathogens. The $16 \mathrm{~s}$ rDNA PCR should thus give a positive signal if a bacterium is present. We retrospectively tested this new version on sonication fluids of patients with a confirmed periprosthetic joint infection (PJI; $n=19$ ) based on the adapted Musculoskeletal Infection Society criteria presented at the 2013 consensus meeting in Philadelphia ${ }^{6}$. We identified the correct pathogens in 9 out of 19 cases with a confirmed PJI (47.4\%) by PCR. In cases where sonication culture was negative or growth was detected only in enrichment broth owing to low inoculum, the multiplex PCR did not identify the pathogen and the universal PCR did not provide additional information, except 1 polymicrobial infection (Table 1A). An explanation for the low sensitivity of the new multiplex PCR Unyvero ITI G2 cartridges could be the PCR detection limit, which is indicated for most pathogens to be between $10^{4}$ and $10^{5}$. However, the low bacterial count is only one of the problems in the diagnosis of bone and joint infections resulting in negative gram staining or no visible bacteria in histopathology ${ }^{7}$. Thus, it is disappointing that the new cartridges of Unyvero ITI G2 with a fast turnaround time were not able to increase sensitivity compared with conventional tissue cultures.

See Tests for septic arthritis diagnosis, page 1588

Personal non-commercial use only. The Journal of Rheumatology Copyright (C) 2018. All rights reserved. 
Table 1A. Comparison of conventional culture techniques and multiplex PCR using standard 50ml concentrated sonicated fluid (SF) in 19 PJI cases.

\begin{tabular}{|c|c|c|c|c|}
\hline Pathogen & Cases, $\mathrm{n}$ & + Sonication Culture & + Multiplex PCR, $50 \mathrm{ml}$ & Comment on the Failure \\
\hline Staphylococcus aureus & 6 & 5 & 3 & $\begin{array}{l}2 \text { missed (positive culture in broth only), } \\
1 \text { missed (negative sonication culture while } \\
\text { taking antibiotics) }\end{array}$ \\
\hline CNS & 4 & 4 & 4 & \\
\hline Enterococcus faecalis & 1 & 1 & 0 & Unknown \\
\hline Morganella morganii & 1 & 1 & 0 & Lack of primer \\
\hline Streptococcus agalactiae & 1 & 1 & 1 & \\
\hline Mycobacterium bovis & 1 & 1 & 0 & Lack of primer \\
\hline Culture negative & 4 & 0 & 0 & \\
\hline Polymicrobial (CNS, Proteus mirabilis) & 1 & 1 (only P. mirabilis) & 1 (CNS, P. mirabilis) & \\
\hline
\end{tabular}

Table $1 B$. Comparison of conventional culture techniques and multiplex PCR using both universal PCR and multiplex primers with standard 50-ml concentrated sonicated fluid (SF) versus higher-concentrated sonicated fluid in 9 PJI and 11 no-PJI cases.

\begin{tabular}{|c|c|c|c|c|}
\hline Microorganism & + Culture & + Universal PCR & $\begin{array}{c}\text { + Multiplex PCR } \\
50 \mathrm{ml} \mathrm{SF}\end{array}$ & $\begin{array}{l}\text { +Multiplex PCR, higher- } \\
\text { volume SF }\end{array}$ \\
\hline Culture-positive PJI, $\mathrm{n}=9$ & 9 & 9 & 8 & 8 \\
\hline Staphylococcus aureus & 3 & 3 & 3 & 3 \\
\hline CNS & 3 & 3 & 3 & 3 \\
\hline Streptococcus agalactiae & 1 & 1 & 1 & 1 \\
\hline Morganella morganii & 1 & 1 & 0 & 0 \\
\hline Polymicrobial (CNS and Proteus mirabilis) & 1 (only P. mirabilis) & 1 & 1 (CNS, P. mirabilis) & 1 (CNS, P. mirabilis) \\
\hline No PJI, $n=11$ & 0 & 0 & 0 & 1 \\
\hline
\end{tabular}

PJI: periprosthetic joint infection; CNS: coagulase-negative staphylococci.

\section{Does concentrating the sonication fluid lead to increased sensitivity of multiplex PCR?}

Morgenstern, et $a l^{5}$ suggested that the use of more concentrated fluids than the current standard 50-ml concentrated fluid may improve diagnostic sensitivity. Aiming to address exactly this question, we used more-concentrated sonication fluid (median fluid $200 \mathrm{ml}$, range $100-500 \mathrm{ml}$ ) to diagnose or exclude a PJI in a small cohort of clear culture-positive and culture-negative PJI. Twenty patients were included [9 PJI, 11 no PJI; median age 65 yrs, range $45-87$ yrs; hip $(n=12)$, knee $(n=4)$, shoulder $(n=4)$ prostheses]. Three $S$. aureus, 3 coagulase-negative staphylococci (CNS), 1 Streptococcus agalactiae, 1 Morganella morganii, and 1 polymicrobial infection were diagnosed. All 9 culturepositive PJI had a positive universal PCR. Multiplex PCR was positive in 8 out of 9 . The M. morganii case was missed because of a lack of specific primers included in the multiplex PCR. The multiplex PCR detected the polymicrobial infection with CNS and Proteus mirabilis despite antibiotic treatment, while culture detected only P. mirabilis. When testing the sonication fluids that had been concentrated more by multiplex PCR, we found no additional information as compared to the standard 50-ml sonication fluid, but a higher numerical signal intensity of the PCR was found (Figure 1).

In all the cases without PJI, universal and multiplex PCR of 50-ml concentrated sonicated fluid were both correctly negative; while higher volume concentrated sonication fluid was positive with Cutibacterium acnes (formerly Propionibacterium acnes) in 1 case (Table 1B). This small study showed that concentrating the current standard 50-ml sonication fluid did not detect additional pathogens by Unyvero multiplex PCR but was false positive with $C$.acnes in one of the cases serving as a control (i.e., without a documented PJI) ${ }^{8}$.

Should conventional cultures remain the gold standard? In the publication by Ivy, et al, authors found 4 microorganisms in 25 cases with culture-negative PJI while investigating synovial fluid with metagenomics shotgun sequencing 9 . These were Salpingoeca rosetta $(\mathrm{n}=2)$, S. aureus, Enterococcus faecalis $(\mathrm{n}=1)$, Finegoldia magna, and Anaerococcus vaginalis $(\mathrm{n}=1)$. In another publication investigating the sonication fluid ${ }^{10}$, microorganisms in culture-negative PJI were found in $31.3 \%$, using metagenomic techniques. Examples of the microorganisms found are Candida albicans, S. aureus, Staphylococcus epidermidis, S. agalactiaeldysgalactiae, Granulicatella adiacens, E. faecalis, and Enterobacter cloacae. The authors found new microorganisms in cases that had been diagnosed as aseptic failures: C. acnes $(\mathrm{n}=2)$, S. aureus $(\mathrm{n}=3)$, and Streptococcus 


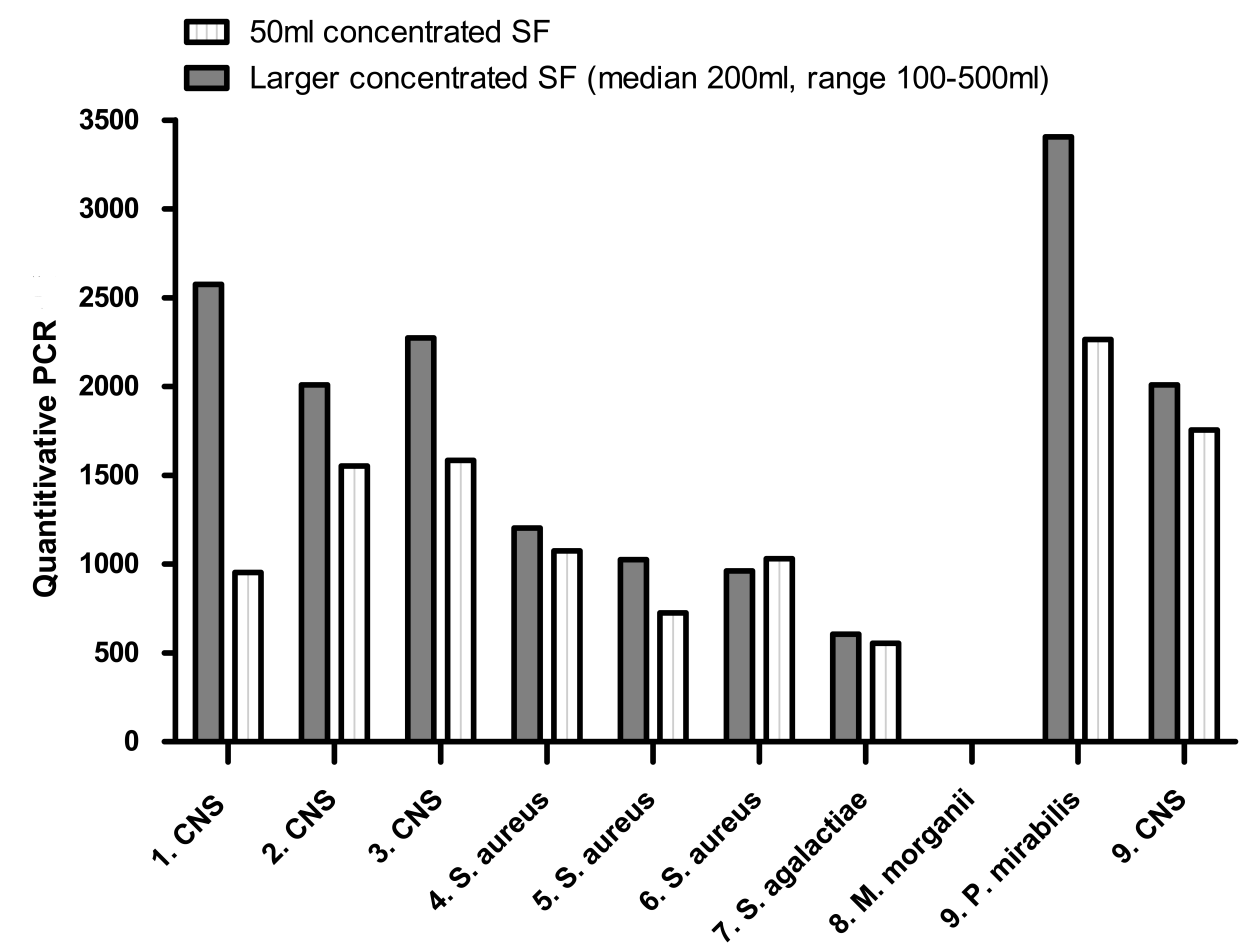

Figure 1. Comparison of multiplex PCR using 50-ml concentrated sonication fluid (SF) or larger SF. Fluorescence density for 10 microorganisms is shown for the 9 patients with proven PJI. Patient 9 had a polymicrobial infection with CNS and Proteus mirabilis. PJI: periprosthetic joint infection; CNS: coagulase-negative staphylococci.

sanguinis $(\mathrm{n}=2)$. All these pathogens are common PJI microorganisms ${ }^{7}$. These 2 publications address an important question and raise many additional ones. What is the gold standard that allows identifying or ruling out a PJI on the one hand and on the other, the causative pathogens in PJI? How many diagnostic repetitions are necessary to definitely rule out a PJI? Are culture-negative bone and joint infections still diseases that do not require antibiotic treatment because of the very low inoculum of typical pathogens that our immune system is able to eliminate? Or should we treat all culture-negative PJI with drugs against bacteria described above as the pathogens that have been found most often to date? More studies are required to assess when treatment is necessary.

\section{YVONNE ACHERMANN ${ }^{\circ}$, MD;}

ANNELIES S. ZINKERNAGEL (D), MD, PhD, MSc,

Division of Infectious Diseases and Hospital Epidemiology,

University Hospital Zurich,

University of Zurich,

Zurich, Switzerland.

Address correspondence to Dr. Y. Achermann, Division of Infectious Diseases and Hospital Epidemiology, University Hospital Zurich, University of Zurich, Raemistrasse 100, CH-8091 Zurich, Switzerland. E-mail: yvonne.achermann@usz.ch

\section{REFERENCES}

1. Achermann Y, Vogt M, Leunig M, Wust J, Trampuz A. Improved diagnosis of periprosthetic joint infection by multiplex PCR of sonication fluid from removed implants. J Clin Microbiol 2010;48:1208-14.

2. Borde JP, Hacker GA, Guschl S, Serr A, Danner T, Hubner J, et al. Diagnosis of prosthetic joint infections using UMD-Universal Kit and the automated multiplex-PCR Unyvero i60 ITI((R)) cartridge system: a pilot study. Infection 2015;43:551-60.

3. Hischebeth GT, Randau TM, Buhr JK, Wimmer MD, Hoerauf A, Molitor E, et al. Unyvero i60 implant and tissue infection (ITI) multiplex PCR system in diagnosing periprosthetic joint infection. J Microbiol Methods 2016;121:27-32.

4. Prieto-Borja L, Rodriguez-Sevilla G, Aunon A, Perez-Jorge C, Sandoval E, Garcia-Canete J, et al. Evaluation of a commercial multiplex PCR (Unyvero i60) designed for the diagnosis of bone and joint infections using prosthetic-joint sonication. Enferm Infecc Microbiol Clin 2017;35:236-42.

5. Morgenstern C, Renz N, Cabric S, Perka C, Trampuz A. Multiplex polymerase chain reaction and microcalorimetry in synovial fluid: can pathogen-based detection assays improve the diagnosis of septic arthritis? J Rheumatol 2018;45:1588-93.

6. Parvizi J, Gehrke T, Chen AF. Proceedings of the International Consensus on Periprosthetic Joint Infection. Bone Joint $\mathrm{J}$ 2013;95-B:1450-2.

7. Tande AJ, Patel R. Prosthetic joint infection. Clin Microbiol Rev 2014;27:302-45.

8. Achermann Y, Kaspar M, Zingg P, Zbinden R, Zinkernagel AS.

Personal non-commercial use only. The Journal of Rheumatology Copyright (c) 2018. All rights reserved. 
Current standard $50 \mathrm{ml}$ sonication fluid concentration is sufficient to detect periprosthetic joint infection pathogens by multiplex PCR.

Poster \#P0712. [Internet. Accessed August 2, 2018.] Available from: www.eccmidlive.org/\#resources/current-standard-50ml-sonicationfluid-concentration-is-sufficient-to-detect-periprosthetic-jointinfection-pathogens-by-multiplex-pcr-72943b10-b9f4-4167-87ac-99 b9320dac71

9. Ivy MI, Thoendel MJ, Jeraldo PR, Greenwood-Quaintance KE, Hanssen AD, Abdel MP, et al. Direct detection and identification of prosthetic joint infection pathogens in synovial fluid by metagenomic shotgun sequencing. J Clin Microbiol 2018 May 30 (E-pub ahead of print).

10. Thoendel M, Jeraldo P, Greenwood-Quaintance KE, Yao J, Chia N, Hanssen $\mathrm{AD}$, et al. Identification of prosthetic joint infection pathogens using a shotgun metagenomics approach. Clin Infect Dis 2018 Apr 10 (E-pub ahead of print).

J Rheumatol 2018;45:1497-1500; doi:10.3899/jrheum.180866 